\title{
LIMIT THEOREMS FOR THE PETERSBURG GAME
}

\section{SÁNDOR CSÖRGÖ ${ }^{1}$ and ROSSITZA DODUNEKOVA ${ }^{2}$}

We determine all possible subsequences $\left\{n_{k}\right\}_{k=1}^{\infty}$ of the positive integers for which the suitably centered and normalized total gain $S_{n_{k}}$ in $n_{k}$ Petersburg games has an asymptotic distribution as $k \rightarrow \infty$, and identify the corresponding set of limiting distributions. We also solve all the companion problems for lightly, moderately, and heavily trimmed versions of the sum $S_{n_{k}}$ and for the respective sums of extreme values in $S_{n_{k}}$.

\section{INTRODUCTION}

A virtually complete and unified theory of the asymptotic distribution of sums of order statistics has been recently worked out in the three papers $[3,4,5]$ with $[2]$ augmenting and rounding off the study in $[3]$. (See, however $[7]$, and also the survey [6|.) Although a number of ad hoc examples are scattered in these papers to illustrate various interesting phenomena, we felt that there was a need for a didactic type of a paper that would illustrate all the limit theorems in the above articles on a single, sufficiently complex but still manageable example which, most importantly, is interesting in its own right. Our attention was drawn to the famous Petersburg game by an interesting paper of Martin-Lö |10]. Since the "timehonored" Petersburg paradox, as Feller (|8|, X. 4) describes it, has been around for 276 years (since the publication of the second edition of Montmort's book in 1713), it is clearly of sufficient interst in itself, and it turns out that it also satisfies the other criteria.

\footnotetext{
1 Partially supported by the Hungarian National Foundation for Scientific Research, Grants $1808 / 86$ and $457 / 88$

${ }^{2}$ Patially supported by the Ministry of Culture, Science and Education of Bulgaria, Contrict No. $16848-F 3$
} 
The 'paradox' has been originally posed by Nicolaus Bernoulli to Montmort in 1713, and the English translation of the nice account of it by Daniel Bernoulli in 1738 is given by Martin-Löf [10]. For a recent historical account and references see Shafer [11]. Following Feller and Martin-Löf, and hence doubling the gain of the Bernoullis, the Petersburg game consists in tossing a fair coin until it falls heads; if this occurs at the $k$-th throw the player receives $2^{k}$ ducates. Hence if $X$ is the gain at a single trial we have $P\left\{X=2^{k}\right\}=2^{-k}$, $k=1,2, \ldots$, and

$$
F(x)=P\{X \leq x\}= \begin{cases}0 & , x<2, \\ 1-2^{|\log x|} & , x \geq 2,\end{cases}
$$

where, and throughout in this paper Log stands for the logarithm to the base $2,\{y\}=$ $\max \{j: j$ integer, $j \leq y\}$ is the usual integer part function and we shall neeed $[y]=$ $\min \{j: j$ integer, $j \geq y\}, y \in \mathbb{R}$.

Let $X_{1}, X_{2}, \ldots$ be independent copies of $X$, the gains at the first, second,... trials, so that $S_{n}=X_{1}+\ldots+X_{n}$ is the total gain in $n$ consecutive Petersburg games. Feller proved that

$$
S_{n} /(n \log n) \rightarrow_{P} 1 \text { as } n \rightarrow \infty
$$

where $\rightarrow P$ denotes convergence in probability and, denoting by $\rightarrow_{D}$ convergence in distribution, Martin-Löf proved that

$$
\frac{S_{2^{k}}}{2^{k}}-k \rightarrow \supset V \quad \text { as } k \rightarrow \infty,
$$

where, if $\Delta_{r}, r=0, \pm 1, \pm 2, \ldots$, are independent Poisson random variables with $E \Delta_{r}=2^{-r}$,

$$
V=\sum_{r=0}^{-\infty}\left(\Delta_{r}-2^{-r}\right) 2^{r}+\sum_{r=1}^{\infty} \Delta_{r} 2^{r}
$$

and hence, with $i$ denoting the imaginary unit,

$$
E e^{i V t}=\exp \left\{\sum_{r=0}^{-\infty} 2^{-r}\left(e^{i 2^{r} t}-1-i 2^{r} t\right)+\sum_{r=1}^{\infty} 2^{-r}\left(e^{i 2^{r} t}-1\right)\right\}
$$


for any $t \in \mathbb{R}$. The limit theorem (1.3) then forms a basis for Martin-Löf to develop a premium formula "which clarifies the 'Petersburg paradox" ", at least when the game is played in blocks of the size $2^{k}, k=1,2, \ldots$, and is very favorable for the casino.

At this point two questions arise. The result in (1.3) means that the Petersburg distribution is in the domain of partial attraction of the infinitely divisible distribution of $V$. Since $V$ is obviously not a stable random variable, it follows from (1.3) and Theorem 10 in [2] that $F$ belongs to the domain of partial attraction of continuum many different types of infinitely divisible distributions and hence there are just as many principle clarifications of the paradox. How different are these from one another? The second question is more practical: What to do if $n_{k}$ games are played, where $2^{k-1}<n_{k}<2^{k}$ ? Some qualitative answers to these questions are indicated by the results for the whole sums $S_{n_{k}}$ in Section 2. It turns out, in particular, that the two questions are more or less the same.

Since the Petersburg game is exciting exactly because of the occasional large single gains in the total gain $S_{n_{k}}$, it is interesting to see the influence of these large gains on the limiting distribution. Let $X_{1, n} \leq \ldots \leq X_{n, n}$ be the order statistics pertaining to the sample $X_{1}, \ldots, X_{n}$. Together with the full sums $S_{n_{k}}$, in Section 2 we also investigate the lightly trimmed sums $S_{n_{k}}(m)=\sum_{j=1}^{n_{k}-m} X_{j, n_{k}}$, where $m \geq 1$ is a fixed integer, while in Section 3 we deal with moderately trimmed sums $S_{n_{k}}\left(m_{k}\right)$, where $m_{k} \rightarrow \infty$ but $r n_{k} / n_{k} \rightarrow 0$ as $k \rightarrow \infty$, and heavily trimmed sums $S_{n}(n-\lfloor\beta n\rfloor)$, where $1 / 2<\beta<1$. But then it is also interesting to see what happens to the extreme sums $S_{n_{k}}-S_{n_{k}}\left(m_{k}\right)$. This is done in Section 4.

The unified method in $[3,4,5]$ and $[2]$ is generally based on the quantile function $Q(s)=\inf \{x: F(x) \geq s\}, 0<s<1$, which from (1.1) presently is

$$
Q(s)=2^{r} \quad \text { if } \quad 1-\frac{1}{2^{r-1}}<s \leq 1-\frac{1}{2^{r}}, r=1,2, \ldots,
$$

and we may put $Q(0)=Q(0+)=2$, or

$$
Q(1-u)=2^{\lceil\log (1 / u)\rceil}, \quad 0<u \leq 1 .
$$


In each of the following three sections we first state all the results of that section, with some discussion when appropriate, and the proofs occupy the remaining part of the section.

\section{FULL AND LIGHTLY TRIMMED SUMS}

The first result restricts the choice of meaningful normalizing and centering sequences. We generally deal with lightly trimmed sums $S_{n_{k}}(m)=\sum_{j=1}^{n_{k}-m} X_{j, n_{k}}$, where $n \geq 0$ is a fixed integer. Since $S_{n}=\sum_{j=1}^{n} X_{j, n}$ for each $n$, we have $S_{n}(0)=S_{n}$, so the special case $m=0$ always corresponds to total gains. Subsequences $\left\{n_{k}\right\}_{k=1}^{\infty}$ of the positive integers are always assumed to go to infinity.

THEOREM 2.1. If for some integer $m_{0} \geq 0$, a subsequence $\left\{n_{k}\right\}_{k=1}^{\infty}$ of the positive integers, and some constants $A_{n_{k}}>0$ and $C_{n_{k}} \in \mathbb{R}$ the sequence $\left(S_{n_{k}}\left(m_{0}\right)-C_{n_{k}}\right) / A_{n_{k}}$ converges in distribution to a non-degenerate random variable $W\left(m_{u}\right)$, then for each subsequence $\left\{n_{k}^{\prime}\right\}_{k=1}^{\infty} \subset\left\{n_{k}\right\}_{k=1}^{\infty}$ and each integer $m \geq 0$ there exist a further subsequence $\left\{n_{k}^{\prime \prime}\right\}_{k=1}^{\infty} \subset\left\{n_{k}^{\prime}\right\}_{k=1}^{\infty}$ and a constant $a=a_{\left\{n^{\prime \prime}\right\}}, 0<a<\infty$, such that $n_{k}^{\prime \prime} / A_{n_{k}^{\prime \prime}} \rightarrow a$ and

$$
W_{n_{k}^{\prime \prime}}(m):=\frac{S_{n_{k}^{\prime \prime}}(m)}{n_{k}^{\prime \prime}}-\left(\left\lceil\log \frac{n_{k}^{\prime \prime}}{m+1}\right\rceil+1-\frac{m+1}{n_{k}^{\prime \prime}} 2^{\left\lceil\log n_{k}^{\prime \prime}\right]}\right) \rightarrow D \frac{1}{a} W(m)+c(m)
$$

as $k \rightarrow \infty$, where $c(m)$ is some constant, $W\left(m_{\dot{u}}\right)$ is as before, and none of the other random variables $W(m)$ is degenerate.

The theorem shows that using the normalizing sequence $\left\{n_{k}\right\}$ and the indicated centering sequence we can achieve all possible limiting types and hence we can restrict attention to these sequences without loss of generality when answering, in particular, the questions posed in the introduction concerning total gains.

Define $W_{n_{k}}=W_{n_{k}}(0)$ as above, replacing $n_{k}^{\prime \prime}$ by $n_{k}$ everywhere. Let us agree that in the diadic expansion of a number $\gamma, 1 / 2<\gamma \leq 1$, given by

$$
\gamma=\sum_{j=1}^{\infty} a_{j} / 2^{j}=\lim _{k \rightarrow \infty} s_{k}, \quad s_{k}=\sum_{j=1}^{k} a_{j} / 2^{j}, \quad k \geq 1,
$$


where $a_{1}=1$ and $a_{j}=0$ or 1 for $j \geq 2$, we exclude all the sequences $\left(1, a_{2}, a_{3}, \ldots\right)$ which contain only a finite number of non-zero elements. Let $E_{1}, E_{2}, \ldots$ be independent exponential random variables with the common expectation 1 and with the Gamma(j) random variables $Y_{j}=E_{1}+\ldots+E_{j}$ as jump-points, consider the standard left-continuous Poisson process

$$
N(s)=\sum_{j=1}^{\infty} I\left(Y_{j}<s\right), \quad s \geq 0,
$$

where $I(\cdot)$ is the indicator function. Also, consider the independent increments

$$
\Delta_{r}(\gamma)=N\left(\gamma 2^{-r+1}\right)-N\left(\gamma 2^{-r}\right), r=0, \pm 1, \pm 2, \ldots, \quad \text { where } \quad \frac{1}{2} \leq \gamma \leq 1
$$

is a fixed number.

THEOREM 2.2. For a given subsequence $\left\{n_{k}\right\}_{k=1}^{\infty}$ of the positive integers the sequence $W_{n_{k}}$ converges in distribution as $k \rightarrow \infty$ if and only if

$$
\lim _{k \rightarrow \infty} \frac{n_{k}}{2^{\left\lceil\log n_{k}\right\rceil}}=\lim _{k \rightarrow \infty} 2^{\log n_{k}-\left\{\log n_{k}\right\rceil}=\gamma \quad \text { for some } \quad \frac{1}{2} \leq \gamma \leq 1
$$

In this case, for any integer $m \geq 0$,

$$
\frac{S_{n_{k}}(m)}{n_{k}}-\left\lceil\log \frac{n_{k}}{m+1}\right\rceil \rightarrow D V_{\gamma}(m),
$$

where, with

$$
\begin{gathered}
\varphi_{\gamma}^{*}(s)=-2^{-[\operatorname{Lug}(s / \gamma)\rfloor}, \quad s>0, \\
V_{\gamma}(m)=1+\frac{1}{\gamma}\left\{\int_{\gamma}^{\infty}(N(s)-s) d \varphi_{\gamma}^{*}(s)+\int_{Y^{\prime m+1}}^{\gamma} N(s) d \varphi_{\gamma}^{*}(s)\right. \\
\left.+m \varphi_{\gamma}^{*}\left(Y_{m+1}\right)-\int_{1}^{m+1} \varphi_{\gamma}^{*}(s) d s-m\right\} .
\end{gathered}
$$

In particular, if $m=0$ then under (2.4),

$$
\frac{S_{n_{k}}}{n_{k}}-\log n_{k} \rightarrow_{D} W_{\gamma}:=V_{\gamma}-\log \gamma
$$


where $V_{\gamma}=V_{\gamma}(0)$ and we have

$$
\begin{aligned}
V_{\gamma}=\frac{1}{\gamma} U_{\gamma} & =\frac{1}{\gamma}\left\{\int_{\gamma}^{\infty}(N(s)-s) d \varphi_{\gamma}^{*}(s)+\int_{0}^{\gamma} N(s) d \varphi_{\gamma}^{*}(s)+\gamma\right\} \\
& =\frac{1}{\gamma}\left\{\sum_{k=1}^{\infty}\left(N\left(\gamma 2^{k}\right)-\gamma 2^{k}\right) 2^{-k}+\sum_{k=0}^{\infty} N\left(\gamma 2^{-k}\right) 2^{k}+\gamma\right\} \\
& =\frac{1}{\gamma}\left\{\sum_{r=0}^{-\infty}\left(\Delta_{r}(\gamma)-\gamma 2^{-r}\right) 2^{r}+\sum_{r=1}^{\infty} \Delta_{r}(\gamma) 2^{r}\right\} .
\end{aligned}
$$

Furthermore, if $\gamma=1 / 2$, then the sequence $n_{k}=n_{k}(1 / 2)=2^{k-1}+1, k=1,2, \ldots$, satisfies (2.4), while if $1 / 2<\gamma \leq 1$, then the sequence

$$
n_{k}=n_{k}(\gamma)=2^{k-1}+a_{2} 2^{k-2}+\ldots+a_{k-1} 2+a_{k}+1, \quad k=1,2, \ldots,
$$

satisfies (2.4), where $a_{2}, a_{3}, \ldots$ are the binary digits in the diadic expansion (2.1).

Martin-Löf's result in (1.3) is obtained by choosing $n_{k}=n_{k}(1)=2^{k}$ in (2.9) and using the third representation of $V=V_{1}=W_{1}=U_{1}$ in (2.8).

It is interesting to note that (2.7) and the general approach in $|3|$, within the framework of which we work in the proofs, at once imply that if $\left\{n_{k}^{(l)}\right\}_{k=1}^{\infty}, l=1, \ldots, r$, are $r$ different sequences satisfying (2.4) with $\gamma=\gamma l, l=1, \ldots, r$, respectively, then

$$
\left(\frac{S_{n_{k}^{(1)}}}{n_{k}^{(1)}}-\log n_{k}^{(1)}, \ldots, \frac{S_{n_{k}^{(r)}}}{n_{k}^{(r)}}-\log n_{k}^{(r)}\right) \rightarrow D\left(W_{\gamma_{1}}, \ldots, W_{\gamma_{r}}\right),
$$

where $W_{\gamma}=\left(U_{\gamma}-\gamma \log \gamma\right) / \gamma$ for any of $\gamma=\gamma_{l}, l=1, \ldots, r$, with $U_{\gamma}$ given by any of the three representations in (2.8) in terms of the same Poisson process. For any $t \in \mathbb{R}$, we have $E \exp \left(i U_{\gamma} t\right)=(E \exp (i V t))^{\gamma}$ with the expression in (1.4) substituted. We see that $U_{\gamma}, 1 / 2 \leq \gamma \leq 1$, determined by (2.10) and explicitly given in (2.8) is a segment of an independent-increment or Lévy process. In fact, $U_{\gamma}, 1 / 2 \leq \gamma \leq 1$, is a segment of a special semi-stable process as described by Lévy [9; Section 58] and we will demonstrate below that

$$
W_{1 / 2}=W_{1}
$$


The above results show that the continuum many infinitely divisible types of distributions that partially attract the Petersburg distribution is given by the set $\left\{G_{\gamma}: 1 / 2 \leq\right.$ $\gamma \leq 1\}$, where $G_{\gamma}(x)=P\left\{W_{\gamma} \leq x\right\}, x \in \mathbb{R}$, and the essential role of the parameter $\gamma$ is in fact to connect the gaps in the special subsequence $\left\{2^{k}\right\}_{k=1}^{\infty}$ of Martin-Lör. We plan to return to this problem by providing a general premium formula that makes successive Petersburg games asymptoticaly fair in a special subsequent note.

In order to get some additional feeling concerning the limiting distribution function $G_{\gamma}$ of $W_{\gamma}$ in (2.7), for each $n=1,2, \ldots$, let $L_{n}(\gamma)$ be the Lévy distance between $G_{\gamma}$ and the approximating distribution function

$$
G_{\gamma}^{(n)}(x)=P\left\{\sum_{j=1}^{n} \frac{1}{\gamma} 2^{-\left\lfloor\log \left(Y_{j} / \gamma\right)\right\rfloor}-\left\lfloor\log \frac{n}{\gamma}\right\rfloor+1-\frac{n}{\left.\gamma^{2} \mid \log (n / \gamma)\right\rfloor}-\log \gamma \leq x\right\}, x \in \mathbb{R} .
$$

Note that, in addition to (2.10), the general probabilistic approach in $|3|$ and the details of the proofs below imply that if $\left\{n_{k}\right\}$ satisfies (2.4) then for any integers $l, m \geq 0$,

$$
\begin{aligned}
& \frac{1}{n_{k}}\left(S_{n_{k}}(l)-n_{k}\left[\log \frac{n_{k}}{l+1}\right], X_{n_{k}-m+1, n_{k}}, \ldots, X_{n_{k}, n_{k}}\right) \\
& \rightarrow_{D}\left(V_{\gamma}(l), \frac{1}{\gamma} 2^{-\left\lfloor\log \left(Y_{m} / \gamma\right)\right\rfloor}, \ldots, \frac{1}{\gamma} 2^{-\left\lfloor\log \left(Y_{1} / \gamma\right)\right\rfloor}\right)
\end{aligned}
$$

as $k \rightarrow \infty$, and so the sum

$$
\sum_{j=1}^{m} \frac{1}{\gamma} 2^{-\left\{\log \left(Y_{j} / \gamma\right)\right\}}
$$

may be looked upon as the asymptotic contribution of the largest $m$ gains to the limiting distribution of the total gain $S_{n_{k}}$ in (2.5). The following result is a corollary to the Theorem in [1] after some straightforward but lengthier calculations not reproduced here.

THEOREM 2.3. For any $\gamma>0$ and any positive number $\rho<1, L_{n}(\gamma)=o\left(n^{-\rho / 2}\right)$ as $n \rightarrow \infty$.

Now we turn to the proofs of the first two theorems. Throughout the paper, all the asymptotic relations are understood to take place as $k \rightarrow \infty$ if not specified otherwise. 
Proof of Theorem 2.1. According to the results in $[3,2]$, the limiting behavior of centered and normalized finitely trimmed sums is by and large determined by the asymptotic behavior of the function

$$
\begin{aligned}
\sigma^{2}(s, t)= & \int_{s}^{1-t} \int_{s}^{1-t}(u \wedge v-u v) d Q(u) d Q(v) \\
= & s Q^{2}(s)+t Q^{2}(1-t)+\int_{s}^{1-t} Q^{2}(u) d u \\
& -\left\{s Q(s)+t Q(1-t)+\int_{s}^{1-t} Q(u) d u\right\}^{2}
\end{aligned}
$$

as $s, t \downarrow 0$, where $u \wedge v=\min (u, v)$ and this well-known equality holds for any $0<s<$ $1-t<1$. Presently we need the function $\sigma^{2}(s)=\sigma^{2}(s, s), 0<s<1 / 2$, for which, substituting (1.5) and (1.6) into (2.13), we obtain by simple calculation that

$$
\sigma^{2}(s)=2^{r} 3-2-(r+1)^{2}, \quad \text { if } \quad \frac{1}{2^{r}} \leq s<\frac{1}{2^{r-1}}, \quad r \geq 2
$$

or, what is the same,

$$
\sigma^{2}(s)=2^{[\log (1 / s)]} 3-2 \cdots([\log (1 / s)]+1)^{2}, \quad 0<s<1 / 2
$$

Hence the asymptotic equality

$$
\sigma(s) \sim \sqrt{3} 2^{[\log (1 / s)] / 2} \text { as } s \downarrow 0
$$

holds, and from the same calculation we also have

$$
\begin{aligned}
\int_{0}^{1-s} Q(u) d u & =\left\lceil\log \frac{1}{s}\right\rceil+1-2^{\lceil\log (1 / s)\rceil} s, \\
& \int_{0}^{1-s} Q^{2}(u) d u=2^{[\log (1 / s)\rceil} 3-2-2^{2\{\log (1 / s)\rceil} s, \quad 0<s \leq 1 .
\end{aligned}
$$

Thus for the sequence

$$
a\left(n_{k}\right)=\sqrt{n_{k}} \sigma\left(1 / n_{k}\right), \quad k=1,2, \ldots
$$


we have by (2.14),

$$
a\left(n_{k}\right) \sim \sqrt{3}{\sqrt{n_{k}}}^{\left[\operatorname{Lic} n_{k}\right] / 2}=\left(3 \frac{2^{\left|\log n_{k}\right|}}{n_{k}}\right)^{1 / 2} n_{k} .
$$

Introducing

$$
\alpha_{k}=\alpha_{n_{k}}=\left\lceil\log n_{k}\right\rceil-\log n_{k} \quad \text { and } \quad \beta_{s}=\log s-\lfloor\log s\rfloor
$$

where, obviously, $0 \leq \alpha_{k}, \beta_{s}<1$ for any $k \geq 1$ and $s>0$, we have

$$
\left[\log \left(n_{k} / s\right)\right\rceil= \begin{cases}\left\lceil\log n_{k}\right\rceil-\lfloor\log s\rfloor & , \text { if } \quad 0 \leq \alpha_{k}+\beta_{s}<1, \\ \left\lceil\log n_{k}\right\rceil-\lfloor\log s\rfloor-1, & \text { if } \quad 1 \leq \alpha_{k}+\beta_{s}<2 .\end{cases}
$$

Whence and from (1.6) and (2.17),

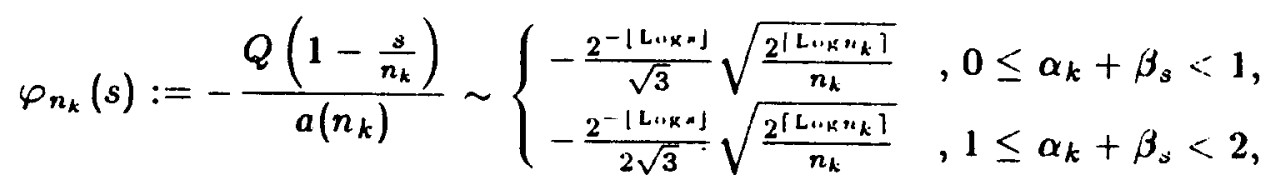

for any fixed $s>0$, where this function plays the role of $\psi^{\prime} \%_{2}\left(n_{k}, s\right)$ in $[3,2]$.

The assumption of the theorem and Theorem 5 in $|3|$ imply that for each subsequence $\left\{n_{k}^{\prime}\right\} \subset\left\{n_{k}\right\}$ there is a further subsequence $\left\{n_{k}^{\prime \prime \prime}\left\{\subset\left\{n_{k}^{\prime}\right\}\right.\right.$ such that both sequences $Q\left(s / n_{k}^{\prime \prime \prime}\right) / A^{n_{k}^{\prime \prime \prime}}$ and $a\left(n_{k}^{\prime \prime \prime}\right) \varphi_{n_{k}^{\prime \prime \prime}}(s) / A_{n_{k}^{\prime \prime \prime}}$ of functions converge weakly (denoted in what follows by $\Rightarrow)$ on $(0, \infty)$ to some finite functions $\varphi_{1}$ and $\varphi_{2}$, respectively, and $a\left(n_{k}^{\prime \prime \prime}\right) / A_{n_{k}^{\prime \prime \prime}} \rightarrow \delta$, where $0 \leq \delta<\infty$. Since, necessarily, $A_{n_{k}} \rightarrow \infty$, it follows from (1.5) that $\varphi_{1} \equiv 0$. But, since

$$
1 \leq 2^{[\log s]} / s<2, \quad s>0
$$

it is clear that if $\delta$ were zero then we would have $\varphi_{2} \equiv 0$, and then by the same Theorem 5 in [3] the limiting random variable $W\left(m_{0}\right)$ would be degenerate. Hence $\delta>0$ and, necessarily, $\varphi_{2} \not \equiv 0$. Therefore, $\varphi_{n_{k}^{\prime \prime \prime}}(\cdot) \Rightarrow \delta^{-1} \varphi_{2}(\cdot)$, and thus by $(2.20)$ there is a further subsequence $\left\{n_{k}^{I V}\right\} \subset\left\{n_{k}^{\prime \prime \prime}\right\}$ such that $a\left(n_{k}^{I V}\right) \varphi_{n_{k}^{\prime \prime}}(\cdot) / n_{k}^{I V} \Rightarrow a \varphi_{2}(\cdot)$ for some $a>0$. If we now define $\tilde{W}_{n_{k}^{\prime l}}(m)$ as $W_{n_{k}^{I V}}(m)$ in the theorem upon replacing $\left[\log n_{k}^{I V}\right\rceil$ by $\left[\log \left(n_{k}^{I V} /(m+1)\right)\right]$, 
then by Theorem 4 in $|3|$ this $\tilde{W}_{n_{k}^{\prime}}(m)$ converges in distribution to $a^{-1} W(m)+\tilde{c}(m)$ for each $m \geq 0$, where in fact the non-degenerate $W(m)$ is represented in a special form, taking into account (2.15) an the fact that $\int_{0}^{1 / n_{k}} Q(u) d u=2 / n_{k} \rightarrow 0$. Now by (2.20),

$$
1-2(m+1)<\frac{m+1}{n_{k}} 2^{\left[\log \left(n_{k} /(m+1)\right)\right]}-\frac{m+1}{n_{k}} 2^{\left[\log n_{k}\right]}<2 \ldots(m+1),
$$

and hence for each $m \geq 0$ we can choose a further subsequence $\left\{n_{k}^{\prime \prime}\right\} \subset\left\{n_{k}^{J V}\right\}$ and a constant $c(m)$, both depending on $m$, such that $W_{n_{k}^{\prime \prime}}(m)$ converges as stated.

We are a little bit sloppy in the defintion of $\varphi_{n_{k}}(\cdot)$ in (2.19). Let $0<\rho_{n_{k}}<1$ be such that $\rho_{n_{k}} \downarrow 0$ and $n_{k} \rho_{n_{k}} \rightarrow 0$, and define $\varphi_{n_{k}}(s)$ by (2.19) if $0<s \leq n_{k}-n_{k} \rho_{n_{k}}$, while if $s>n_{k}-n_{k} \rho_{n_{k}}$ then set $\varphi_{n_{k}}(s)=-Q\left(\rho_{n_{k}}\right) / a\left(n_{k}\right)$. Now $\varphi_{n_{k}}(\cdot)$ is a negative, non-decreasing function on the whole $(0, \infty)$ with $\varphi_{n_{k}}(0)=\varphi_{n_{k}}(0+)=-\infty$.

LEMMA 2.4. For a given subsequence $\left\{n_{k}\right\}_{k=1}^{\infty}$ the functions $\varphi_{n_{k}}(\cdot)$ converge weakly to some finite function given on $(0, \infty)$ if and only if condition (2.4) holds. In this case,

$$
\varphi_{n_{k}}(\cdot) \Rightarrow \varphi_{\gamma}(\cdot):=\frac{1}{\sqrt{3 \gamma}} \varphi_{\gamma}^{*}(\cdot)=-\frac{1}{\sqrt{3 \gamma}} 2^{-\lfloor\log (\cdot / \gamma)\rfloor}
$$

with the right-continuous function $\varphi_{\gamma}^{*}(\cdot)$ taken from (2.6).

Proof. In the notation of (2.18), condition (2.4) is equivalent to

$$
\alpha_{k}=\alpha_{n_{k}} \rightarrow \alpha \text { for some } 0 \leq \alpha \leq 1
$$

in which case

$$
\gamma=2^{-\alpha}
$$

First we prove the necessity of (2.22). Suppose that weak convergence takes place but (2.22) is not true. Then for two subsequences $\left\{n_{k}^{\prime}\right\}$ and $\left\{n_{k}^{\prime \prime}\right\}$ we have $\underline{\alpha}_{k}=\alpha_{n_{k}^{\prime}} \rightarrow \underline{\alpha}=$ 
$\liminf \alpha_{k}$ and $\bar{\alpha}_{k}=\alpha_{n_{k}^{\prime \prime}} \rightarrow \bar{\alpha}=\limsup \alpha_{k}$, where $0 \leq \underline{\alpha}<\bar{\alpha} \leq 1$. Clearly, we can find a continuity point $s>0$ of the limiting function such that for $\beta_{s}$ defined in (2.18) we have $\underline{\alpha}<1-\beta_{s}<\bar{\alpha}$, and hence for all $k$ large enough $\underline{\alpha}_{k}<1 \cdots \beta_{s}<\bar{\alpha}_{k}$ or, what is the same, $\underline{\alpha}_{k}+\beta_{s}<1$ and $\bar{\alpha}_{k}+\beta_{s}>1$. Thus from (2.19),

$$
\varphi_{n_{k}^{\prime}}(s) \rightarrow-\sqrt{\frac{2^{\alpha}}{3}} 2^{-\lfloor\text {Ligg } s\rfloor} \text { and } \varphi_{n_{k}^{\prime \prime}}(s) \rightarrow-\frac{1}{2} \sqrt{\frac{2^{\bar{\alpha}}}{3}} 2^{-\lfloor\log s\rfloor}
$$

Equating the two limits, we obtain $\bar{\alpha}=2+\underline{\alpha}$, which is impossible.

Now we consider sufficiency. Using (2.4), (2.22), and (2.23), from (2.19) we obtain that if $0<\alpha<1$, or equivalently, $1 / 2<\gamma<1$, then

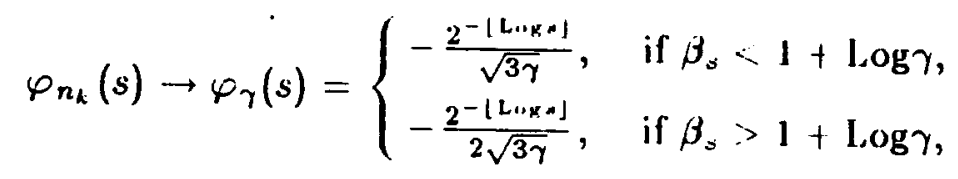

if $\gamma=1$, then

$$
\varphi_{n_{k}}(s) \rightarrow \varphi_{1}(s)=-\frac{2^{-\lfloor\text {Ligg } s \mid}}{\sqrt{3}}, \quad s>0
$$

and if $\gamma=1 / 2$, then

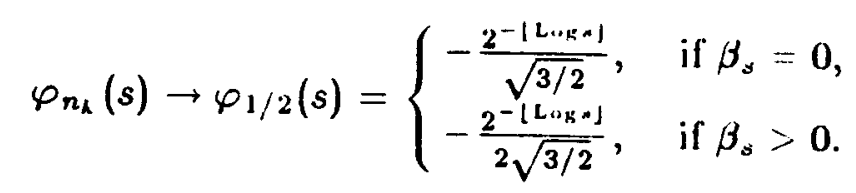

When $\gamma=1$ or $\gamma=1 / 2$, it is obvious that $\varphi_{\gamma}(\cdot)$ can be written in the form claimed in (2.21). An elementary argument shows that this is also true when $1 / 2<\gamma<1$. (Or course, if $1 / 2<\gamma<1$ then there is no convergence in general at those $s>0$ for which $\beta_{s}=1+\log \gamma$. These are the jump-points $s=\gamma 2^{k}, k=0, \pm 1, \pm 2, \ldots$. See also (2.27) below.)

Proof of Theorem 2.2. Before starting the actual proof, we note that for any sequence $\left\{n_{k}\right\}$ by (2.14) and (2.20) we have

$$
\frac{\sigma^{2}\left(h / n_{k}\right)}{\sigma\left(1 / n_{k}\right)} \sim \frac{1}{h} \frac{n_{k}}{2^{\left\lceil\log n_{k}\right\rceil}} \frac{2^{\left\lceil\log \left(n_{k} / h\right)\right\rceil}}{n_{k} / h}<\frac{2}{h}, \quad h>0
$$


and hence

$$
\lim _{h \rightarrow \infty} \limsup _{k \rightarrow \infty} \sigma\left(h / n_{k}\right) / \sigma\left(1 / n_{k}\right)=0 .
$$

Also, whatever is the sequence $\left\{n_{k}\right\}$ like, it follows from (2.20) that any subsequence $\left\{n_{k}^{\prime}\right\} \subset\left\{n_{k}\right\}$ contains a further subsequence $\left\{n_{k}^{\prime \prime}\right\} \subset\left\{n_{k}^{\prime}\right\}$ such that $(2.4)$ is satisfied along $\left\{n_{k}^{\prime \prime}\right\}$ and with some $\gamma=\gamma_{\left\{n^{\prime \prime}\right\}}, 1 / 2 \leq \gamma \leq 1$. Then by (2.17), $a\left(n_{k}^{\prime \prime}\right) / n_{k}^{\prime \prime} \rightarrow \sqrt{3 / \gamma}$ and hence by Lemma 2.4, for $\varphi_{n_{k}}^{*}(\cdot)=a\left(n_{k}\right) \varphi_{n_{k}}(\cdot) / n_{k}$ we have

$$
\varphi_{n_{a}^{* \prime \prime}}(\cdot) \Rightarrow \frac{1}{\gamma} \varphi_{\gamma}^{*}(\cdot)
$$

Since $Q\left(\left(s / n_{k}\right)+\right) / n_{k} \rightarrow 0$ for all $s>0$, this last weak convergence, (2.24) and (2.15) imply by an application of Theorem $1^{*}$ in $|2|$ (which is an augmented form of Theorem 1 in $|3|$ ) that for any $m \geq 0$ in $W_{n_{k}^{\prime \prime}}(m)$ of Theorem 2.1 we have

$$
\begin{aligned}
& W_{n_{k}^{\prime \prime}}(m) \rightarrow D \frac{1}{\gamma} V_{0, m}\left(0, \varphi_{. \gamma}^{\cdot}, 0\right) \\
& :=\frac{1}{\gamma}\left\{\int_{Y_{m+1}}^{\infty}(N(s)-s) d \rho_{\gamma}^{*}(s) \cdot \int_{1}^{Y_{m+1}^{\prime}} s d \varphi_{\gamma}^{*}(s)\right. \\
& \left.+m \varphi_{\gamma}^{*}\left(Y_{m+1}\right)-\int_{1}^{m+1} \varphi_{\gamma}^{*}(s) d s-\varphi_{\gamma}^{\prime}(1)\right\} .
\end{aligned}
$$

Now if $W_{n_{k}}=W_{n_{k}}(0)$ converges in distribution, then its subsequental limits are all of the form $V_{0,0}\left(0, \varphi_{\gamma}^{*}, 0\right) / \gamma$ having an infinitely divisible distribution. Since this representation, being a special case of the general representation in Theorem 3 in $|3|$, is unique, it follows that $\gamma$ must be the same for all the above subsequences $\left\{n_{k}{ }^{\prime \prime}\right.$, and hence (2.4) is necessarily satisfied.

Suppose now (2.4). Then for any $m \geq 0$, the above convergence of $W_{n_{k}}(m)$ takes place along the original $\left\{n_{k}\right\}$. It follows then that the left side of $(2.5)$ converges in distribution to

$$
\frac{1}{\gamma} V_{0, m}\left(0, \varphi_{\gamma}^{*}, 0\right)+1-\frac{m+1}{\gamma},
$$


and since by easy manipulation one can see that

$$
\begin{aligned}
\int_{Y_{m+1}}^{\infty}(N(s)-s) d \varphi_{\gamma}^{*}(s)-\int_{1}^{Y_{m+1}} s d \varphi_{\gamma}^{*}(s) \\
\quad=\int_{\gamma}^{\infty}(N(s)-s) d \varphi_{\gamma}^{*}(s)+\int_{Y_{m+1}}^{\gamma} N(s) d \varphi_{\gamma}^{*}(s)+\int_{\gamma}^{1} s d \varphi_{\gamma}^{*}(s)
\end{aligned}
$$

(in fact, regardless of a meaningful function $\varphi$ replacing the present $\varphi_{\gamma}^{*}$ ) and, separating the cases $\gamma=1 / 2$ and $1 / 2<\gamma \leq 1$, that

$$
\int_{\gamma}^{1} s d \varphi_{\gamma}^{*}(s)-\varphi_{\gamma}^{*}(1)=1, \quad 1 / 2 \leq \gamma \leq 1
$$

(see (2.27) below), it is clear that the limit in (2.25) is the same as $V_{\gamma}(m)$ in (2.5). Thus (2.5) holds, and since under (2.4),

$$
\left\lceil\log n_{k}\right\rceil-\log n_{k} \rightarrow-\log \gamma
$$

the convergence in (2.7) and the first representation in (2.8) also follow. Furthermore, since the function $\varphi_{\gamma}^{*}$ in $(2.6)$ can be written as

$$
\varphi_{\gamma}^{*}(s)=-2^{-k}, \quad \gamma 2^{k} \leq s<\gamma 2^{k+1}, \quad k=0, \pm 1, \pm 2, \ldots
$$

the second representation in (2.8) follows from the first by carrying out the integration.

To prove the last equation in $(2.8)$, note that by simple computation,

$$
\begin{aligned}
& \sum_{k=1}^{j}\left(N\left(\gamma 2^{k}\right)-\gamma 2^{k}\right) 2^{-k}+\sum_{k=0}^{j} N\left(\gamma 2^{-k}\right) 2^{k}+\gamma \\
& =\sum_{k=0}^{j-1}\left(\left[N\left(\gamma 2^{k+1}\right)-N\left(\gamma 2^{k}\right)\right]-\gamma 2^{k}\right) 2^{-k}+\left[N\left(\gamma 2^{j}\right)-\gamma 2^{j}\right] 2^{-j} \\
& \quad+\sum_{k=1}^{j}\left[N\left(\gamma 2^{-k+1}\right)-N\left(\gamma 2^{-k}\right)\right] 2^{k}+N\left(\gamma 2^{-j}\right) 2^{j+1}
\end{aligned}
$$

for each $j \geq 1$. Since the second term goes to zero almost surely by the law of large numbers as $j \rightarrow \infty$ and the fourth term is almost surely zero for all $j$ large enough, the last line of (2.8) follows upon letting $j \rightarrow \infty$. 
Finally, it is trivial that $n_{k}(1 / 2)=2^{k-1}+1$ satisfies $(2.4)$ with $\gamma=1 / 2$. Also, if $1 / 2<\gamma \leq 1$, then it is plain that $\left[\log n_{k}(\gamma)\right\rceil=k$ for the sequence $n_{k}(\gamma)$ in $(2.9)$ and hence, in the notation of (2.1),

$$
\frac{n_{k}(\gamma)}{2_{\left.\log n_{k}(\gamma)\right\}}}=\frac{n_{k}(\gamma)}{2^{k}}=s_{k}+2^{-k} \rightarrow \gamma
$$

The theorem is completely proved.

Proof of (2.11). From the third representation in (2.8) we obtain

$$
\begin{aligned}
V_{1 / 2}= & \sum_{k=0}^{\infty}\left(\left[N\left(2^{k}\right)-N\left(2^{k-1}\right)\right]-2^{k-1}\right) \frac{1}{2^{k-1}}+\sum_{k=1}^{\infty}\left[N\left(\frac{1}{2^{k}}\right)-N\left(\frac{1}{2^{k+1}}\right)\right] 2^{k+1} \\
= & \sum_{r=0}^{\infty}\left(\left[N\left(2^{r+1}\right)-N\left(2^{r}\right)\right]-2^{r}\right) \frac{1}{2^{r}}+\sum_{r=1}^{\infty}\left[N\left(\frac{1}{2^{r-1}}\right)-N\left(\frac{1}{2^{r}}\right)\right] 2^{r} \\
& \left.+\left(\mid N(1)-N\left(\frac{1}{2}\right)\right]-\frac{1}{2}\right) 2-\left[N(1)-N\left(\frac{1}{2}\right) \mid 2\right. \\
= & V_{1}-1,
\end{aligned}
$$

which is the same as (2.11).

\section{MODERATELY AND HEAVILY TRIMMED SUMS}

Let $\left\{n_{k}\right\}_{k=1}^{\infty}$ and $\left\{m_{n_{k}}\right\}_{k=1}^{\infty}$ be two subsequences of the positive integers such that

$$
m_{k} \rightarrow \infty \text { and } m_{n_{k}} / n_{k} \rightarrow 0
$$

and consider the moderately trimmed sums

$$
S_{n_{k}}\left(m_{n_{k}}\right)=\sum_{j=1}^{n_{k}-m_{\prime_{k}}} X_{j, n_{k}} .
$$

The first result is an analogue of Theorem 2.1 in the present setting. 
THEOREM 3.1. If (3.1) is satisfied and for some constants $A_{n_{k}}>0$ and $C_{n_{k}} \in$ $\mathbb{R}$ the sequence $\left(S_{n_{k}}\left(m_{n_{k}}\right)-C_{n_{k}}\right) / A_{n_{k}}$ converges in distribution to a non-degenerate random variable $W$, then for each subsequence $\left\{n_{k}^{\prime}\right\}_{k=1}^{\infty}<\left\{n_{k}\right\}_{k=1}^{\infty}$ there exist a further subsequence $\left\{n_{k}^{\prime \prime}\right\}_{k=1}^{\infty} \subset\left\{n_{k}^{\prime}\right\}_{k=1}^{\infty}$ and a constant $a=a_{\left\{n^{\prime \prime}\right\}}, 0<a<\infty$, such that $\left(n_{k}^{\prime \prime} / \sqrt{m_{n_{k}^{\prime \prime}}}\right) / A_{n_{k}^{\prime \prime}} \rightarrow a$ and

$$
W_{n_{k}^{\prime \prime}}:=\sqrt{m_{n_{k}^{\prime \prime}}}\left\{\frac{S_{n_{k}^{\prime \prime}}\left(m_{n_{k}^{\prime \prime}}\right)}{n_{k}^{\prime \prime}}-\left(\left[\log \frac{n_{k}^{\prime \prime}}{m_{n_{k}^{\prime \prime}}}\right]+1-\frac{m_{n_{k}^{\prime \prime}}}{n_{k}^{\prime \prime}} 2^{\left\{\log \left(n_{k}^{\prime \prime} / m_{n_{k}^{\prime \prime}}\right) \mid\right.}\right)\right\} \rightarrow D \frac{1}{a} W^{\prime}+c \text {, }
$$

as $k \rightarrow \infty$, where $c$ is some constant.

Again we see that it is enough to deal with centering and norming sequences of the given special form. Define now $W_{n_{k}}$ as in the above theoren, replacing $n_{k}^{\prime \prime}$ by $n_{k}$. Set $\varepsilon_{n_{k}}=\left\lceil\log \left(n_{k} / m_{n_{k}}\right)\right\rceil-\log \left(n_{k} / m_{n_{k}}\right)$.

THEOREM 3.2. For a given subsequence $\left\{n_{k}\right\}_{k=1}^{\infty}$ of the positive integers and a sequence $\left\{m_{n_{k}}\right\}_{k=1}^{\infty}$ satisfying (3.1) the sequence $W_{n_{k}}$ converges in distribution as $k+\infty$ if and only if one of the following three mutually exclusive conditions holds :

$$
\begin{gathered}
\sqrt{m_{n_{k}}}\left(1-2^{-\varepsilon_{n_{k}}}\right) \rightarrow \infty \quad \text { and } \sqrt{m_{n_{k}}}\left(1-2^{1-\varepsilon_{u_{k}}}\right) \rightarrow-\infty \\
\sqrt{m_{n_{k}}}\left(1-2^{1-\varepsilon_{n_{k}}}\right) \rightarrow v \text { for some } \quad-\infty<v \leq 0 \\
\sqrt{m_{n_{k}}}\left(1-2^{-\varepsilon_{n_{k}}}\right) \rightarrow u \text { for some } 0 \leq u<\infty
\end{gathered}
$$

as $k \rightarrow \infty$.

If (3.2) holds then

$$
\frac{\sqrt{m_{n_{k}}}}{B\left(n_{k}\right)}\left\{\frac{S_{n_{k}}\left(m_{n_{k}}\right)}{n_{k}}-\left(\left\lceil\log \frac{n_{k}}{m_{n_{k}}}\right\rceil+1-B^{2}\left(n_{k}\right)\right)\right\} \rightarrow D \sqrt{3} Z
$$

as $k \rightarrow \infty$, where $Z$ is a standard normal random variable and

$$
B\left(n_{k}\right)=\left(\frac{2^{\left\lceil\log \left(n_{k} / m_{n_{k}}\right)\right\rceil}}{n_{k} / m_{n_{k}}}\right)^{1 / 2}
$$


If (3.3) holds then, as $k \rightarrow \infty$,

$$
\sqrt{m_{n_{k}}}\left\{\frac{S_{n_{k}}\left(m_{n_{k}}\right)}{n_{k}}-\log \frac{n_{k}}{m_{n_{k}}}\right\} \rightarrow D \sqrt{6}\left\{Z+\max \left(0, v+Z_{2}\right)\right\}
$$

where $\left(Z, Z_{2}\right)$ is a bivariate normal vector with mean vector zero, $E Z^{2}=E Z_{2}^{2}=1$, and $E Z Z_{2}=-\sqrt{2 / 3}$

If (3.4) holds then, as $k \rightarrow \infty$,

$$
\sqrt{m_{n_{k}}}\left\{\frac{S_{n_{k}}\left(m_{n_{k}}\right)}{n_{k}}-\log \frac{n_{k}}{m_{n_{k}}}\right\} \rightarrow D \sqrt{3}\left\{Z+\max \left(0,-u-Z_{2}\right)\right\}
$$

where $\left(Z, Z_{2}\right)$ is a bivariate normal vector with mean vector zero, $E Z^{2}=E Z_{2}^{2}=1$, and $E Z Z_{2}=-1 / \sqrt{3}$.

Furthermore, the pair of sequences $\left(n_{k}, m_{n_{k}}\right)$ where $m_{n_{k}}$ is an arbitrary sequence of positive integers such that $m_{n_{k}} \rightarrow \infty$, as $k \rightarrow \infty$, and

$$
n_{k}=m_{n_{k}}\left(2^{k-1}+a_{2} 2^{k-2}+\ldots+a_{k-1} 2+a_{k}\right), \quad k=1,2, \ldots
$$

where $a_{2}, a_{3}, \ldots$ are the binary digits in the diadic expansion (2.1) of any fixed number $1 / 2<\gamma<1$, satisfies (3.2).

The pair $\left(n_{k}, m_{n_{k}}\right)$ given for any $k=1,2, \ldots$ by

$$
n_{k}=2^{2 q_{k}-1}+2^{2 q_{k} r_{k}-1}\left\{\lfloor-v / \sqrt{2}\rfloor+\sum_{j=1}^{2 q_{k}-r_{k}-1} v_{j} 2^{-j}\right\}, \quad m_{n_{k}}=2^{2 r_{k}+1}-1
$$

where $q_{k}$ and $r_{k}$ are arbitrary positive integers such that

$$
q_{k} \rightarrow \infty, \quad r_{k} \rightarrow \infty, \quad \text { and } \quad q_{k}-r_{k} \rightarrow \infty \text { as } k \rightarrow \infty
$$

and $v_{1}, v_{2}, \ldots$ are the binary digits in the diadic expansion of $-v / \sqrt{2}-\lfloor-v / \sqrt{2}\rfloor$ satisfies (3.3) with $v<0$, and the pair $\left(n_{k}, m_{n_{k}}\right)$ given by

$$
n_{k}=2^{3 q_{k}-1}+2^{3 q_{k}-2 r_{k}}, \quad m_{n_{k}}=2^{3 r_{k}+1}-1, \quad k=1,2, \ldots
$$


with $q_{k}$ and $r_{k}$ satisfying (3.8), satisfies (3.3) with $v=0$.

Finally, with $q_{k}$ and $r_{k}$ satisfying (3.8), the pair $\left(n_{k}, m_{n_{k}}\right)$ given for any $k=1,2, \ldots$ by

$$
n_{k}=2^{2 q_{k}}-2^{2 q_{k}-r_{k}}\left\{|u|+\sum_{j=1}^{2 q_{k}-r_{k}} u_{j} 2^{-j}\right\}, \quad m_{n_{k}}=2^{2 r_{k}}+1,
$$

where $u_{1}, u_{2}, \ldots$ are the binary digits in the diadic expansion of $u-\lfloor u\rfloor$, satisfies (3.4) with $u>0$, while the pair $\left(n_{k}, m_{n_{k}}\right)$ given for any $k=1,2, \ldots$ by

$$
n_{k}=2^{q_{k}-1}, \quad m_{n_{k}}=2^{r_{k}}+1
$$

satisfies (3.4) with $u=0$.

We note that when talking about a binary expansion of a number in $(0,1)$ in the above theorem we always use the convention following (2.1), but we also assume that all the binary digits of zero are zero.

It is interesting to point out the fact that the sequence $B\left(n_{k}\right)$ in $(3.5)$, for which by (2.20) we have

$$
1 \leq B\left(n_{k}\right)<\sqrt{2}
$$

does not in general converge under (3.2). In fact, if

$$
0<\liminf _{k \rightarrow \infty} \varepsilon_{n_{k}} \leq \limsup _{k \rightarrow \infty} \varepsilon_{n_{k}}<1
$$

then we always have (3.2).

The last result of this section is for heavily trimmed sums

$$
S_{n}(n-\lfloor\beta n\rfloor)=\sum_{j=1}^{\lfloor\beta n\rfloor} X_{j, n}, \quad \text { where } \quad \frac{1}{2}<\beta<1 .
$$

It is a special case of a half-sided version of Theorem 5 in $[4]$ easily stated for an underlying distribution that is concentrated on the positive half line. 
THEOREM 3.3. (i) If $-\log (1-\beta)$ is not an integer, then

$$
\sqrt{n}\left\{\frac{\sum_{j=1}^{\lfloor\beta n\rfloor} X_{j, n}}{n}-\mu(\beta)\right\} \rightarrow_{D} \sigma_{1}(0, \beta) Z
$$

as $n \rightarrow \infty$, where $Z$ is a standard normal random variable,

$$
\mu(\beta)=2-[\log (1-\beta)\rceil-2(1-\beta) 2^{-[\log (1-\beta)]}>0
$$

and

$$
\sigma_{1}(0, \beta)=\left(6\left\{2^{-|\log (1-\beta)|}-1\right\}+2[\log (1-\beta)\}-\{\log (1-\beta)]^{2}\right)^{1 / 2} .
$$

(ii) If $-\log (1-\beta)$ is an integer, then

$$
\sqrt{n}\left\{\frac{\sum_{j=1}^{\lfloor\beta n} X_{j, n}}{n}-\log \frac{1}{1-\beta}\right\} \rightarrow_{D} \sigma_{2}(0, \beta) Z+(1-\beta)^{-1 / 2} \max \left(0,-Z_{2}\right),
$$

where $\left(Z, Z_{2}\right)$ is a bivariate normal vector with mean vector zero, $E Z^{2}=1, E Z_{2}^{2}=\beta$, and

$$
E Z Z_{2}=-\{2 \beta+(1-\beta) \log (1-\beta)\} /\left\{1+2 \beta-(1-\beta)(1-\log (1-\beta))^{2}\right\}^{1 / 2}
$$

and where $\sigma_{2}(0, \beta)=\left(\left\{1+2 \beta-(1-\beta)(1-\log (1-\beta))^{2}\right\} /(1-\beta)\right)^{1 / 2}$.

Part (i) of this theorem leads to an interesting modification of the Petersburg game. Suppose the casino and the player agree to play $n$ consecutive Petersburg games so that the largest $n-\mid \beta n\rfloor$ principal gains will not be payed to the player, where $-\log (1-\beta)$ is not an integer. Since we have

$$
P\left\{\sum_{j=1}^{\lfloor\beta n\rfloor} X_{j, n}>n \mu(\beta)\right\} \rightarrow \frac{1}{2} \text { and } P\left\{\sum_{j=1}^{\lfloor\beta n\rfloor} X_{j, n}<n \mu(\beta)\right\} \rightarrow \frac{1}{2}
$$

as $n \rightarrow \infty$, the fair premium for the player to pay to the casino for playing this sequence of $n$ games is $n \mu(\beta)$ if $n$ is large enough. Of course the casino can also determine a different premium formula from part (i) of the theorem to raise unfairly its fair chance $1 / 2$.

Proof of Theorem 3.1. Presently we need the norming sequence

$$
a_{n_{k}}\left(m_{n_{k}}\right)=\sqrt{n_{k}} \sigma\left(m_{n_{k}} / n_{k}\right)
$$


instead of the one in (2.16), for which by $(2.14)$,

$$
a_{n_{k}}\left(m_{n_{k}}\right) \sim \sqrt{3} \sqrt{n_{k}} 2^{\left[\log \left(n_{k} / m_{n_{k}}\right)\right] / 2}=\left(3 \frac{2^{\left[\log \left(n_{k} / m_{n_{k}}\right)\right]}}{n_{k} / m_{n_{k}}}\right)^{1 / 2} \frac{n_{k}}{\sqrt{m_{n_{k}}}} .
$$

Also, we use $\varepsilon_{k}=\varepsilon_{n_{k}}$ in the formulation of Theorem 3.2, that is

$$
\varepsilon_{k}=\varepsilon_{n_{k}}=\left\lceil\log \left(n_{k} / n_{n_{k}}\right)\right\rceil-\log \left(n_{k} / m_{n_{k}}\right)
$$

and introduce the functions, that play the role of $\psi_{2, n_{k}}$ in $|4|$,

$$
\psi_{n_{k}}(x)=\frac{\sqrt{m_{n_{k}}}}{a_{n_{k}}\left(m_{n_{k}}\right)}\left\{Q\left(1-\frac{m_{n_{k}}}{n_{k}}+x \frac{\sqrt{m_{n_{k}}}}{n_{k}}\right)-Q\left(1-\frac{m_{n_{\ell}}}{n_{k}}\right)\right\},|x| \leq \frac{\sqrt{m_{n_{k}}}}{2},
$$

on $\mathbb{R}$ by setting $\psi_{n_{k}}(x)=\psi_{n_{k}}\left(-\sqrt{m_{n_{k}}} / 2\right)$ if $x<-\sqrt{m_{n_{k}}} / 2$ and $\psi_{n_{k}}(x)=\psi_{n_{k}}\left(\sqrt{m_{n_{k}}} / 2\right)$ if $x>\sqrt{m_{n_{k}}} / 2$. We can separate the values of these functions according to the three alternatives $d_{k}(x) \geq 1,0 \leq d_{k}(x)<1$, and $d_{k}(x)<0$, where, with $\varepsilon_{k}$ from (3.11), $d_{k}(x)=\varepsilon_{k}+\log \left(1-x / \sqrt{m_{n_{k}}}\right)$, and after showing that

$$
\begin{gathered}
d_{k}(x) \geq 1 \quad \text { if and only if } x<0 \text { and } \sqrt{m_{n_{k}}}\left(1-2^{1-\varepsilon_{k}}\right) \geq x, \\
0 \leq d_{k}(x)<1 \quad \text { if and only if } \sqrt{m_{n_{k}}}\left(1-2^{1-\varepsilon_{k}}\right) \leq x \leq \sqrt{m_{n_{k}}}\left(1-2^{-\varepsilon_{k}}\right), \\
d_{k}(x)<0 \text { if and only if } x>0 \text { and } x>\sqrt{m_{n_{k}}}\left(1-2^{-\varepsilon_{k}}\right),
\end{gathered}
$$

we obtain by (1.6) and (2.14) that for any fixed $x \in \mathbb{R}$,

$$
\psi_{n_{k}}(x) \sim \begin{cases}-\frac{1}{\sqrt{3}} \frac{1}{2} B\left(n_{k}\right) & , \text { if } x<0 \text { and } \sqrt{m_{n_{k}}}\left(1-2^{1-\varepsilon_{k}}\right) \geq x \\ 0 & , \text { if } \sqrt{m_{n_{k}}}\left(1-2^{1-\varepsilon_{k}}\right)<x \leq \sqrt{m_{n_{k}}}\left(1-2^{-\varepsilon_{k}}\right) \\ \frac{1}{\sqrt{3} B\left(n_{k}\right)} & , \text { if } x>0 \text { and } \sqrt{m_{n_{k}}}\left(1-2^{-\varepsilon_{k}}\right)<x\end{cases}
$$

where $B\left(n_{k}\right)$ is as in (3.5), satisfying (3.9).

Using the assumption of the theorem and the converse half of Theorem 3 in $[4]$ (as formulated in Section 3 of [6]; see also the end of Section 1 in [5]), we see that for each $\left\{n_{k}^{\prime}\right\} \subset\left\{n_{k}\right\}$ there is a further $\left\{n_{k}^{\prime \prime \prime}\right\} \subset\left\{n_{k}^{\prime}\right\}$ such that $\psi_{n_{k}^{\prime \prime \prime}}(\cdot) \Rightarrow \psi(\cdot)$ for some finite function $\psi(\cdot)$ on $\mathbb{R}$ and

$$
a_{n_{k}^{\prime \prime \prime}}\left(m_{n_{k}^{\prime \prime \prime}}\right) / A_{n_{k}^{\prime \prime \prime}} \rightarrow \delta, \quad 0<\delta<\infty
$$


(The case $\delta=0$ can be ruled out again because then the limit would be degenerate.) Thus, using also (3.10), (3.9), (2.15), Theorem 1 in [4], and the convergence of types theorem, it is now routine to see that there exist a subsequence $\left\{n_{k}^{\prime \prime}\right\} \subset\left\{n_{k}^{\prime \prime \prime}\right\}$ and constants $0<a<\infty$ and $c \in \mathbb{R}$ such that all the statements of the theorem hold true.

Again, before the proof of Theorem 3.2 we need a technical lemma, paralel to Lemma 2.4. The proof of this lemma uses ideas very similar to those in the proof of Lemma 2.4 but, of necessity, is lengthier and more complicated. In the interest of saving space, it is omitted here.

LEMMA 3.4. For a given $\left\{n_{k}\right\}_{k=1}^{\infty}$ and $\left\{m_{n_{k}}\right\}_{k=1}^{\infty}$ satisfying (3.1) the functions $\psi_{n_{k}}$ converge weakly to some non-decreasing left-continuous function $\psi$ on $\mathbb{R}$, satisfying $\psi(0) \leq 0$ and $\psi(0+) \geq 0$ if and only if one of the conditions (3.2), (3.3) or (3.4) holds. If (3.2) holds then

$$
\psi_{n_{k}}(x) \rightarrow 0 \text { for every } x \in \mathbb{R}
$$

If (3.3) holds then

$$
\varepsilon_{n_{k}} \rightarrow 1, \quad \psi_{n_{k}}(\cdot) \Rightarrow \psi_{v}(\cdot) \quad \text { on } \mathbb{R}, \quad \text { and } B\left(n_{k}\right) \rightarrow \sqrt{2} \text {, }
$$

where

If (3.4) holds then

$$
\psi_{v}(x)= \begin{cases}-1 / \sqrt{6}, & x \leq v, \\ 0 & , \quad x>v .\end{cases}
$$

$$
\varepsilon_{n_{k}} \rightarrow 0, \quad \psi_{n_{k}}(\cdot) \Rightarrow \psi^{u}(\cdot) \quad \text { on } \mathbb{R}, \quad \text { and } \quad B\left(n_{k}\right) \rightarrow 1 \text {, }
$$

where

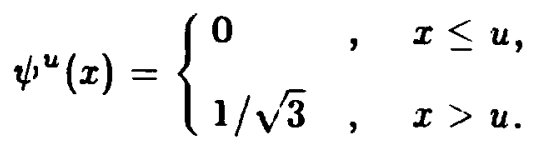


Proof of Theorem 3.2. First we consider the three suficiency statements. If (3.2) holds, then the statement follows by a direct application of Theorem 1 in $[4]$ on account of (3.13) of Lemma 3.4, using (3.10).

If (3.3) holds then by Lemma 3.4 we have (3.14). Using the norming factor $a_{n_{k}}^{+}=$ $n_{k} / \sqrt{m_{n_{k}}}$ instead of $a_{n_{k}}\left(m_{n_{k}}\right) \sim \sqrt{6} n_{k} / \sqrt{m_{n_{k}}}$, the latter obtained from (3.10) and (3.14), the left side of (3.6) converges in distribution to

$$
\sqrt{6}\left\{Z+\int_{0}^{-Z_{2}} \psi_{v}(x) d x\right\}=\sqrt{6}\left\{Z+\max \left(0, v+Z_{2}\right)\right\}
$$

by Theorem 1 in $[4]$, where the covariance $E Z Z_{2}=-\sqrt{2 / 3}$ is obtained by the fact that presently

$$
r_{2, n_{k}}:=-\frac{\sqrt{m_{n_{k}}}}{a_{n_{k}}\left(m_{n_{k}}\right)} \int_{0}^{1-m_{n_{k}} / n_{k}} s d Q(s) \sim-\frac{B^{2}\left(n_{k}\right)}{\sqrt{6}}
$$

which follows by integrating by parts and using formulae (1.6) and (2.15), and hence by (3.14) we obtain $-\sqrt{2 / 3}$ in the limit.

Finally, if (3.4) holds then the proof is exactly the same as above, replacing the use of (3.14) by that of (3.15) and noting that presently we use $a_{n_{k}}^{*}$ instead of $a_{n_{k}}\left(m_{n_{k}}\right) \sim$ $\sqrt{3} n_{k} / \sqrt{m_{n_{k}}}$, that the limit of the left side of (3.7) is, again by Theorem 1 in $|4|$, now

$$
\sqrt{3}\left\{Z+\int_{0}^{-Z_{2}} \psi^{u}(x) d x\right\}=\sqrt{3}\left\{Z+\max \left(0,-u-Z_{2}\right)\right\}
$$

and that (3.16) is still true with $\sqrt{6}$ replaced by $\sqrt{3}$, and hence by (3.15) we now obtain $r_{2, n_{k}} \rightarrow E Z Z_{2}=-1 / \sqrt{3}$.

Now we turn to necessity. Using (3.10), (3.9), and the converse result referred to in the proof of Theorem 3.1, it follows that if $W_{n_{k}}$ converges in distribution then for any subsequence $\left\{n_{k}^{\prime}\right\} \subset\left\{n_{k}\right\}$ there is a further subsequence $\left\{n_{k}^{\prime \prime}\right\} \subset\left\{n_{k}^{\prime}\right\}$ such that for $\psi_{n_{k}}$ in (3.12) we have $\psi_{n_{k}^{\prime \prime}}(\cdot) \Rightarrow \psi(\cdot)$ on $\mathbb{R}$ for some appropriate limiting function $\psi$. But then by Lemma 3.4 we must have exactly one of (3.2), (3.3), and (3.4) along $\left\{n_{k}^{\prime \prime}\right\}$. Applying now 
the already proved sufficency results along $\left\{n_{k}^{\prime \prime}\right\}$, we get one of the three possible limiling behavior. Since, obviously, no two of the three limiting random variables in (3.5), (3.6), and (3.7) can be equal in distribution for any choice of $v \leq 0$ and $u \geq 0$, we see that these subsequential limits must be the same, exactly one of those in (3.5), (3.6), or (3.7). Since the subsequence $\left\{n_{k}^{\prime}\right\} \subset\left\{n_{k}\right\}$ was arbitrary, the necessity statement follows.

The proof of the last statements of the theorem, that is, that the stated constructions for (3.2), (3.3), and (3.4) to hold are indeed valid is elementary but all together extremely tedious and lengthy. We challenge the interested reader to check what we state.

\section{EXTREME SUMS}

Again and throughout this last section, $\left\{n_{k}\right\}_{k=1}^{\infty}$ and $\left\{m_{n_{k}}\right\}_{k=1}^{\infty}$ will be two subsequences of the positive integers satisfying (3.1), and we are interested in the exreme sums

$$
\boldsymbol{E}_{n_{k}}\left(m_{n_{k}}\right)=S_{n_{k}}-S_{n_{k}}\left(m_{n_{k}}\right)=\sum_{j=n_{k}-m_{n_{k}}+1}^{n_{k}} X_{j, n_{k}}=\sum_{j=1}^{m n_{k}} X_{n_{k}+1-j, n_{k}},
$$

the sums of the largest $m_{n_{k}}$ gains. Following the pattern of the preceding two sections, first we state the following.

THEOREM 4.1. If for some constants $A_{n_{k}}>0$ and $C_{n_{k}} \in \mathbb{R}$ the sequence $\left(E_{n_{k}}\left(m_{n_{k}}\right)-C_{n_{k}}\right) / A_{n_{k}}$ converges in distribution to a non-degenerate random variable $R$, then for each subsequence $\left\{n_{k}^{\prime}\right\}_{k=1}^{\infty} \subset\left\{n_{k}\right\}_{k=1}^{\infty}$ there exist a further subsequence $\left\{n_{k}^{\prime \prime}\right\}_{k=1}^{\infty} \subset\left\{n_{k}^{\prime}\right\}_{k=1}^{\infty}$ and a constant $a=a_{\left\{n^{\prime \prime}\right\}}, 0<a<\infty$, such that $n_{k}^{\prime \prime} / A_{n_{k}^{\prime \prime}} \rightarrow a$ and

$$
R_{n_{k}^{\prime \prime}}:=\frac{E_{n_{k}^{\prime \prime}}\left(m_{n_{k}^{\prime \prime}}\right)}{n_{k}^{\prime \prime}}-r_{n_{k}^{\prime \prime}}\left(m_{n_{k}^{\prime \prime}}\right) \rightarrow D \frac{1}{a} R+c
$$

as $k \rightarrow \infty$, where

$$
\begin{aligned}
r_{n_{k}}\left(m_{n_{k}}\right)= & \left(2^{2\left\lceil\log n_{k}\right\rceil-\left\lfloor\log n_{1}\right\rfloor-1}-2^{\left\lceil\log n_{k}\right\rceil}\right) \frac{1}{n_{k}}+\left\lceil\log n_{k}\right\rceil-\left\lceil\log \left(n_{k} / m_{n_{k}}\right)\right\rceil \\
& +2^{\left\lceil\log \left(n_{k} / m_{n_{k}}\right)\right\rceil} \frac{m_{n_{k}}}{n_{k}}
\end{aligned}
$$

and $c$ is some constant. 
Again, let $R_{n_{k}}$ be as above, replacing $n_{k}^{\prime \prime}$ by $n_{k}$. Our last result is the following.

THEOREM 4.2. For a given subsequence $\left\{n_{k}\right\}_{k=1}^{\sim}$ of positive integers and an arbitrary subsequence $\left\{m_{n_{k}}\right\}_{k=1}^{\infty}$ of positive integers satisfying (3.1) the sequence $R_{n_{k}}$ converges in distribution if and only if the sequence $\left\{n_{k}\right\}_{k=1}^{\infty}$ satisfies condition (2.4) of Theorem 2.2 with some $1 / 2 \leq \gamma \leq 1$. In this case,

$$
\left.\frac{E_{n_{k}}\left(m_{n_{k}}\right)}{n_{k}}-\left(\log m_{n_{k}}+\left\{2^{\left\lceil\log \left(n_{k} / m_{n_{k}}\right)\right.}\right\rceil \frac{m_{n_{k}}}{n_{k}}+\log \frac{n_{k}}{m_{n_{k}}}-\left\lceil\log \frac{n_{k}}{m_{n_{k}}}\right\rceil\right\}\right) \rightarrow_{D} W_{\gamma_{1}}
$$

where $W_{\gamma}$ is the limiting random variable appearing in (2.7).

Of course, the special constructions of $\left\{n_{k}\right\}$ in and above (2.9) for condition (2.4) are still valid, and we emphasize that $\left\{m_{n_{k}}\right\}$ satisfying (3.1) is completely arbitrary. The normalizing sequence $\left\{n_{k}\right\}$ is the same for full sums and extreme sums. This is of course entirely natural having (2.12). The results in Section 2 show that the Petersburg distribution function $F$ is stochastically compact (cf. [3]) and we also see that the largest gain $X_{n, n}$ is also stochastically compact, with all possible limiting distributions given in (2.12). So the Petersburg game exhibits the phenomenon discussed in Corollary 12 in $|3|$.

It is interesting to observe the generally non-convergent oscillatory term in the centering sequence in Theorem 4.2 , for which, if log stands for the natural logarithm, it is easy to see that

$$
\frac{1}{\log 2}-\log \frac{1}{\log 2} \leq\left\{2^{\left\lceil\log \left(n_{k} / m_{n_{k}}\right)\right\rceil} \frac{m_{n_{k}}}{n_{k}}+\log \frac{n_{k}}{m_{n_{k}}}-\left\lceil\log \frac{n_{k}}{m_{n_{k}}}\right\rceil\right\} \leq 1
$$

Proof of Theorem 4.1. Recalling the notation in (2.13), we now need to know the asymptotic behavior of $\sigma^{2}\left(1-m_{n_{k}} / n_{k}, 1 / n_{k}\right)$. Using (1.5), (1.6), (2.13), and both formulae in (2.15) yield

$$
\sigma^{2}\left(1-m_{n_{k}} / n_{k}, 1 / n_{k}\right) \sim 2^{\left[\log n_{k}\right]_{3}}
$$


for any $m_{n_{k}}$ satisfying (3.1). Hence, instead of the special norming sequence $\sqrt{n_{k}} \sigma(1-$ $\left.m_{n_{k}} / n_{k}, 1 / n_{k}\right)$ designed for extreme surns in $|5|$, we can use $a\left(n_{k}\right)$ belonging to whole sums, since by (2.17),

$$
\sqrt{n_{k}} \sigma\left(1-m_{n_{k}} / n_{k}, 1 / n_{k}\right) \sim\left(3 \frac{2^{\left|\log n_{k}\right|}}{n_{k}}\right)^{1 / 2} n_{k} \sim a\left(n_{k}\right) .
$$

According to $[5]$, here we need the following variants of the functions $\varphi_{n_{k}}$ and $\psi_{n_{k}}$ in the proofs of Theorems 2.1 and 3.1:

$$
\bar{\varphi}_{n_{k}}(s):= \begin{cases}\frac{-Q\left(\left(1-\frac{k}{n_{k}}\right)-\right)+Q\left(\left(1-\frac{1}{n_{k}}\right)-\right)}{a\left(n_{k}\right)}, & 0<s \leq n_{k}-n_{k} \rho_{n_{l}}, \\ \frac{-Q\left(\rho_{n_{k}}-\right)+Q\left(\left(1-\frac{1}{n_{k}}\right)-\right)}{a\left(n_{k}\right)} & , \quad n_{k}-n_{k} \rho_{n_{k}}<s<\infty,\end{cases}
$$

where $\rho_{n_{k}}$ is the sequence introduced before Lemma 2.4 , and

$$
\bar{\psi}_{n_{k}}(x)= \begin{cases}\frac{\sqrt{m_{n_{k}}}}{a\left(n_{k}\right.}\left\{Q\left(\left(1-\frac{m_{n_{k}}}{n_{k}}\right)-\right)-Q\left(\left(1-\frac{m_{n_{k}}}{n_{k}}-x \frac{\sqrt{m_{n_{k}}}}{n_{k}}\right)-\right)\right\} & ,|x|<\frac{\sqrt{m_{m_{k}}}}{2}, \\ \bar{\psi}_{n_{k}}\left(-\sqrt{m_{n_{k}}} / 2\right) & , x<\frac{v^{m_{n_{k}}}}{2}, \\ \bar{\psi}_{n_{k}}\left(\sqrt{m_{n_{k}}} / 2\right) & , \frac{\sqrt{m_{n_{k}}}<x .}{2}<.\end{cases}
$$

By (2.17), (2.20), (3.10), (3.12), and (4.2) it is obvious that there is a positive constant $C>0$ such that $\psi_{n_{\AA}}(x) \leq C / \sqrt{m_{n_{k}}}$ for any $x \in \mathbb{R}$, and hence by (3.1),

$$
\bar{\psi}_{n_{k}}(x) \rightarrow 0, \quad x \in \mathbb{R}
$$

On the other hand, with $\varphi_{n_{k}}$ given in (2.19), we have

$$
\bar{\varphi}_{n_{k}}(s)=\varphi_{n_{\Lambda}}(s-)-\varphi_{n_{k}}(1-), \quad s>0 .
$$

Also, by a computation similar to that leading to (4.1) we obtain that for any positive numbers $l_{n_{k}}$ such that $l_{n_{k}} \rightarrow \infty$ and $l_{n_{k}} / m_{n_{k}} \rightarrow 0$ we have

$$
\sigma^{2}\left(1-l_{n_{k}} / n_{k}, m_{n_{k}} / n_{k}\right) \sim 2^{\left[\left.\operatorname{Lug}\left(n_{k} / l_{n_{k}}\right)\right|_{3}\right.}
$$

and from (2.15) and (1.6),

$$
\frac{1}{n_{k}} Q\left(\left(1-\frac{1}{n_{k}}\right)-\right)+\int_{1-m_{n_{k}} / n_{k}}^{1-1 / n_{k}} Q(u) d u=r_{n_{k}}\left(m_{n_{k}}\right) .
$$


After all these preliminaries we are ready now to prove the theorem. Using the assumption of it, (4.2), (4.3), (4.4), (2.19), and (2.20), an application of Theorem 2 in $[5]$ shows that for any $\left\{n_{k}^{\prime}\right\} \subset\left\{n_{k}\right\}$ there is a further subsequence $\left\{n_{k}^{\prime \prime \prime}\right\} \subset\left\{n_{k}^{\prime}\right\}$ such that

$$
\bar{\psi}_{n_{k}^{\prime \prime \prime}}(x) \rightarrow 0, \quad x \in \mathbb{R}, \quad \bar{\varphi}_{n_{k}^{\prime \prime \prime}}(\cdot) \Rightarrow \bar{\varphi}(\cdot) \quad \text { on }(0, \infty) \quad \text { and } \quad a\left(n_{k}^{\prime \prime \prime}\right) / A_{n_{k}^{\prime \prime \prime}} \rightarrow \delta
$$

where $\bar{\varphi}$ is a finite function and $\delta>0$. Hence an application of Theorem 1 in $[5]$, and the convergence of types theorem provide a subsequence $\left\{n_{k}^{\prime \prime}\right\} \subset\left\{n_{k}^{\prime \prime \prime}\right\}$ along which the statements hold.

Proof of Theorem 4.2. First we note that by (4.1) and (4.5),

$$
\sigma\left(1-l_{n_{k}} / n_{k}, m_{n_{k}} / n_{k}\right) / \sigma\left(1-m_{n_{k}} / n_{k}, 1 / n_{k}\right), 0
$$

for any sequences $\left\{n_{k}\right\}$ and $\left\{m_{n_{k}}\right\}$ satisfying (3.1) and for any sequence $\left\{l_{n_{k}}\right\}$ of positive numbers such that $l_{n_{k}} \rightarrow \infty$ and $l_{n k} / m_{n_{k}} \rightarrow 0$.

Also, for each fixed $s>0$, by (4.4) and (2.19) we have

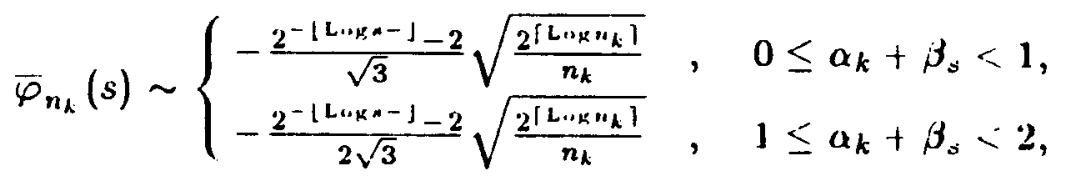

where $\alpha_{k}$ and $\beta_{s}$ are as in (2.19), and clearly the proof of Lemma 2.4 implies that $\bar{\varphi}_{n_{k}}(\cdot)$ converges weakly on $(0, \infty)$ to some finite function if and only if condition (2.4) holds. In particular,

$$
\bar{\varphi}_{n_{k}}(\cdot) \Rightarrow \frac{1}{\sqrt{3 \gamma}}\left(\bar{\varphi}_{\gamma}^{*}(\cdot)-\bar{\varphi}_{\gamma}^{*}(1)\right) \text { on }(0, \infty) \text { if and only if }(2.4) \text { holds, }
$$

where $\bar{\varphi}_{\gamma}^{*}$ is the left-continuous version of $\varphi_{\gamma}^{*}$ given in (2.6).

Assume now that $R_{n_{k}}$ converges in distribution along the given $\left\{n_{k}\right\}$. Consider an arbitrary subsequence $\left\{n_{k}^{\prime}\right\} \subset\left\{n_{k}\right\}$. Then, obviously, there is a further subsequence $\left\{n_{k}^{\prime \prime}\right\} \subset\left\{n_{k}^{\prime}\right\}$ such that condition $(2.4)$ is satisfied for some $1 / 2 \leftrightharpoons \gamma \leq 1$ along this $\left\{n_{k}^{\prime \prime}\right\}$. But then, using (4.3), (4.7), and (4.8) along $\left\{n_{k}^{\prime \prime}\right\}$, it follows from Theorem 1 in $|5|$ that

$$
R_{n_{k}^{\prime \prime}} \rightarrow D \frac{1}{\gamma} \bar{V}_{0, \omega}\left(0, \bar{\varphi}_{\gamma}^{*}, 0\right):=\frac{1}{\gamma}\left\{\int_{Y_{1}}^{\infty}(\bar{N}(s)-s) d \bar{\varphi}_{\gamma}^{*}(s)-\int_{1}^{Y_{1}} s d \bar{\varphi}_{\gamma}^{*}(s)-\bar{\varphi}_{\gamma}^{*}(1)\right\}
$$


where

$$
\bar{N}(s)=\sum_{j=1}^{\infty} I\left(Y_{j} \leq s\right), \quad s \leq 0
$$

is the right-continuous version of the left-continuous Poisson process given in (2.2) and where we use the convention that the symbol $\int_{x}^{y}$ means $\int_{(x, y)}$ whenever we integrate with respect to a left-continuous function, just as the convention that $\int_{x}^{y}$ means $\int_{(x, y)}$ whenever we integrate with respect to a righ-continuous function has been tacitly used throughout the paper. Using these conventions it is easy to see that

$$
\bar{V}_{0, u}\left(0, \bar{\varphi}_{\gamma}^{*}, 0\right)=V_{0, u}\left(0, \varphi_{\gamma}^{*}, 0\right)=\int_{Y_{1}}^{\infty}(N(s)-s) d \varphi_{\gamma}^{*}(s)-\int_{1}^{Y_{1}} s d \varphi_{\gamma}^{*}(s)-\varphi_{\gamma}^{*}(1),
$$

and hence, using the special case $m=0$ of the formula right below $(2.25)$, we see that (4.9) is the same as

$$
R_{n_{k}^{\prime \prime}} \rightarrow D V_{\gamma}^{\prime}
$$

where $V_{\gamma}$ is as in (2.7) and (2.8). At the same time, just as in the proof of 'Theorem 2.2, we see that $\gamma$ must be unique for all these subsequences $\left\{n_{k}^{\prime \prime}\right\}$, and since $\left\{n_{k}^{\prime}\right\}$ was an arbitrary subsequence of $\left\{n_{k}\right\}$, condition (2.4) must hold along $\left\{n_{k}\right\}$, and hence we also have

$$
R_{n_{k}}=\frac{E_{n_{k}}\left(m_{n_{k}}\right)}{n_{k}}-r_{n_{k}}\left(m_{n_{k}}\right) \rightarrow D V_{\gamma}
$$

Conversely, if condition (2.4) is satisfied then, by (4.3), (4.7), and (4.8), Theorem 1 in [5] implies (4.10). But under (2.4) the first term of $r_{n_{k}}\left(m_{n_{k}}\right)$, given in Thorem 4.1, converges to zero, and hence by (2.26) we obtain

$$
\frac{E_{n_{k}}\left(m_{n_{k}}\right)}{n_{k}}-\left(\log n_{k}-\left\lceil\log \left(n_{k} / m_{n_{k}}\right)\right]+2^{\left\lceil\log \left(n_{k} / m_{n_{k}}\right) \mid\right.} \frac{m_{n_{k}}}{n_{k}}\right) \rightarrow D W_{\gamma}
$$

which by adding and subtracting $\log n_{n_{\ell}}$ in the centering sequence is clearly equivalent to the stated convergence in the theorem. 


\section{REFERENCES}

[1] S. CSÖRGŐ, An extreme-sum approximation to infinitely divisible law's without a normal component. In: Probability on Vector Spaces IV. (S. Cambanis and A. Weron, eds.) pp. 000-000. Lecture Notes in Mathematics 0000, Springer, Berlin, 1989.

[2] S. CSÖRGÖ, A probabilistic approach to domains of partial attraction. Adv. in Appl. Moth. To appear.

[3] S. CSÖRGÖ, E. HAEUSLER, and D. M. MASON, A probabilistic approach to the asymptotic distribution of sums of independent, identically distributed random variables. Adv. in Appl. Math. 9(1988), 259-333.

[4] S. CSÖRGÖ, E. HAEUSLER, and D. M. MASON, The asymptotic distribution of trimmed sums. Ann. Probab. 16(1988), 672-699.

[5] S. CSÖRGÖ, E. HAEUSLER, and D. M. MASON, The asymptotic distribution of extreme sums. Ann. Probab. To appear.

[6] S. CSÖRGÖ, E. HAEUSLER, and D. M. MASON, The quantile - transform - empirical - process approach to limit theorems for sums of order statistics. In: Sums, Trimmed Sums, and Extremes (M. G. Hahn, ed.), pp. 000-000. Birkhäuser, Basel, 1990. To appear.

[7] S. CSÖRGŐ and D. M. MASON, Intermediate sums and stochastic compactness of maxima. In preparation.

[8] W. FELLER, An Introduction to Probability Theory and its Applications, Vol.1. Wiley, New York, 1950.

[9] P. LÉVY, Théorie de l'addition des variables aléatoires, $2^{\text {nd }}$ ed. Gauthier - Villars, Paris, 1954.

[10] A. MARTIN - LÖF, A limit theorem which clarifies the 'Petersburg paradox'. J. Appl. Probab. 22(1985), 634-643. 
[11] G. SHAFER, The St. Petersburg paradox. In: Encyclopedia of Statistical Sciences, Vol. 8 (S. Kotz, N. L. Johnson, and C. B. Read, eds.), pp. 865-870. Wiley, New-York, 1988.

Sándor Csörgő

Bolyai Institute

University of Szeged

Aradi vértanúk tere 1

H.6720 Szeged

Hungary
Rossitza Dodunekova

Department of Probability and Statistics University of Sofia

P. O. Box 979

1090 Sofia

Bulgaria 